\title{
The Challenges Towards Energy-Efficient Cognitive Radio Networking
}

\author{
Ahmed Khattab \\ Electronics and Electrical Communications Department \\ Cairo University \\ Giza, Egypt \\ akhattab@ieee.org
}

\author{
Magdy A. Bayoumi \\ The Center for Advanced Computer Studies (CACS) \\ University of Louisiana at Lafayette \\ Lafayette, LA, USA \\ mab@cacs.louisiana.edu
}

\begin{abstract}
The Cognitive Radio (CR) technology is envisioned as a primary solution to the spectrum scarcity problem in future wireless communication networks. Cognitive radio networking allows the exploitation of unutilized spectrum in an opportunistic manner, and thereby, improves the efficiency of the spectrum utilization. Cognitive radio Networks (CRNs) provide wireless connectivity via heterogeneous wireless architectures and dynamic spectrum access techniques. The enabling technologies and the benefits of $C R$ and CRNs have received significant research and industry interest. However, the energy efficiency of such technologies has received far less interest. In this paper, we shed the light on the move towards energy-efficient CRN that fits the global vision of green communication networks.
\end{abstract}

Keywords-cognitive radio; cognitive radio networks; green communications; sustainable development

\section{INTRODUCTION}

The Information and Communications Technologies (ICT) infrastructure has been recently shown to contribute with approximately $3 \%$ of the worldwide energy consumption and $2 \%$ of the global carbon dioxide $\left(\mathrm{CO}_{2}\right)$ emission [1], [2]. This implies that the ICT carbon dioxide emission is approximately equivalent to that of airplanes and 1/4 that of automobiles worldwide. Such a fact has directed the ICT community to a paradigm shift towards green communications wherein energy efficiency is an essential requirement for the development and realization of communications systems [3].

Cognitive Radio Networks (CRNs) are foreseen as the future wireless ICT that exploit dynamic and opportunistic spectrum access strategies to provide wireless connectivity. CRNs are considered as the key technology to alleviate the severe spectrum underutilization and provide a solution for spectrum scarcity [4]. The enabling component of CRNs and opportunistic spectrum access is the cognitive radio (CR). A $\mathrm{CR}$ is a wireless device that senses the surrounding radio environments and opportunistically access unutilized spectrum bands based on the activities of the surrounding primary licensed networks [5]. Intensive research efforts have been carried out on the different ideas and architecture of cognitive radio networking over the past decade [4]. However, the energy efficiency of CRNs has received far less interest.

In 2009, CR and CRNs have been claimed as the enabling technologies for green ICT [1] due to their awareness of the radio frequency (RF) activities over different frequency bands in the surrounding environment. Hence, energy-efficient CRNs are highly desirable for ICT sustainable development. In this paper, we present an overview of the energy challenges of CRNs and how to tackle them in the move towards green ICT.

The rest of the paper is organized as follows. In Section II, we present an overview of the CR cognition cycle. We

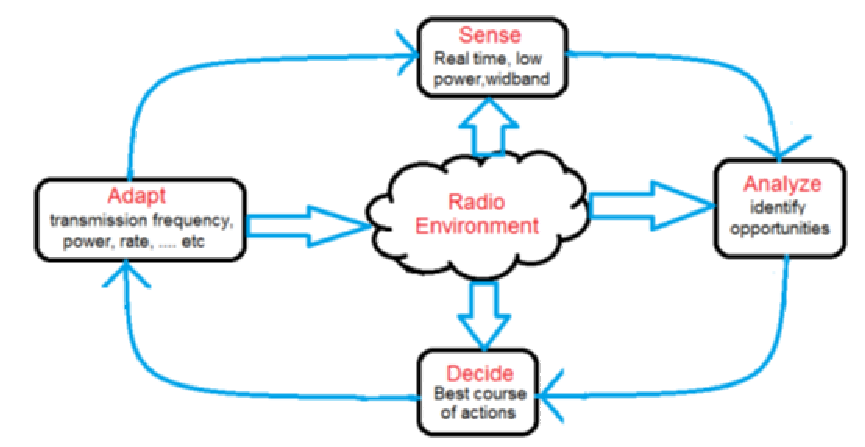

Fig. 1. Cognition cycle of a cognitive radio.

discuss the main energy challenges of CRNs in Section III. We present the main approaches in the move towards a green CRN paradigm in Section IV, and conclude the paper in Section V.

\section{Cognitive Radio Cognition CyCle}

The operation of a CR is described through the cognition cycle defined by Mitola in his seminal work and depicted in Figure 1. The CR cognition cycle has four main steps:

- Spectrum Sensing: Spectrum sensing is the process by which a CR measures the radio frequency (RF) activities due to the ongoing transmissions over different spectrum bands in its surrounding environment. During the sensing process, the CR captures the parameters related to such bands (e.g., cumulative power levels, user activities, etc.). Spectrum sensing is one of the most critical functions of a cognitive radio as it provides the awareness of the spectrum usage in the surrounding environment [6]. However, spectrum sensing is the most power-hungry component of the cognition cycle as will be explained in Section III.

- Spectrum Analysis: Spectrum analysis is to process the sensed RF information of different spectra in order to identify the existence of spectral opportunities in the surrounding radio environment. A spectral opportunity is conventionally defined as "a band of frequencies that are not being used by the primary user of that band at a particular time in a particular geographic area" [6]. However, such a definition is not general enough as it covers only three dimension of the spectrum space: frequency, time, and space. Other dimensions of a given spectrum, such as the coding dimension in spread spectrum systems, can also be exploited.

- Spectrum Access Decisions: Based on the analysis of the sensed spectral information, a CR decides the 
set of transmission actions and the values of the transceiver parameters to be configured for an upcoming transmission. The set of transceiver parameters to be decided depends on the underlying transceiver architecture. Examples of the action set can include which spectrum is more favorable for an upcoming transmission, the time instant a transmission over a certain band should start, the maximum transmission power, the modulation rate, the spread spectrum hopping scheme, the angle of arrival for directional transmissions, and the number and identity of the antennas to be used in multi-antenna systems, etc.

- CR Reconfigurability: A key feature that distinguishes a CR from a traditional radio, and completes the cognition cycle depicted in Figure 1, is its ability to re-tune its transceiver parameters on the fly based on its assessment of the surrounding RF environment. A CR transceiver should be more flexible than just configuring simple parameters such as the transmission rate and power in order to be able to exploit emerging spectral opportunities over a wider spectrum range. For instance, a CR must be able to configure the transmission bandwidth to adapt to spectral opportunities of different sizes, and accordingly choose the appropriate communication protocol.

\section{Cognitive Radio EnERgy Challenges}

A CR must make real-time decisions about which spectrum bands to sense, when, and for how long. The sensed spectrum information must be sufficient enough for the $\mathrm{CR}$ to reach accurate conclusions regarding the radio environment. Furthermore, spectrum sensing must be fast in order to track the temporal variations of the radio environment. Such requirements of spectrum sensing does not only put stringent requirements on the hardware implementation of CRs in terms of the sensing bandwidth, the processing speed, and the RF circuitry, but also represent the main energy-hungry component of a CR. In what follows, we discuss the energy consumption challenges in $\mathrm{CR}$ transceivers and CRNs which are summarized in Figure 2.

\section{A. CR Hardware High Energy Consumption}

There exists many factors that significantly contributes to the energy consumption of spectrum sensing in cognitive radios. First, the cognitive transceiver hardware is required to achieve sufficiently high sensitivity for a wide spectrum (e.g., multiple $\mathrm{GHz}$ ) while accurately detecting diverse and frequency-dependent primary signals at different received power levels. This places severe requirements on the sensitivity, linearity, and dynamic range of the circuitry in the RF front-end, and more specifically, the antennas, power amplifiers and the analog-to-digital conversion units [4], [6]. Furthermore, the processing power requirements of the signal processing units that analyze the sensed spectrum are very high in order for the cognitive radio to make a decision with relatively low delay. Note that the environment is dynamic and the interference is modulated both by the bursty traffic of primary users and channel fading. Such stringent performance and processing requirements necessitates the CR transceiver circuity be power-hungry, and hence, not green.

For instance, the energy consumption of the high power amplifier (HPA) of a radio transceiver is typically $70 \%$ of the total energy consumed during transmission - regardless the communication standard [1]. If the power consumption of the HPA in CR terminal can be efficiently managed, e.g., via Peak to Average Power Ratio (PAPR) sensor, it can significantly reduce the energy consumption of the CR. This requires the design of adaptive HPA that can be tuned on the fly to amplify different signals that belong to different communication standards [1].

\section{B. Listen-Before-Talk Idle Sensing}

Existing spectrum sensing techniques depend on detecting the activities of the primary transmitters. Primary transmitter detection schemes are generally classified into matched filter detection, energy detection, feature detection, and interference temperature measurement. Currently, there does not exist any practical way that allows CR terminals to measure the interference at nearby primary network receivers [4], [6] since primary users are passive and do not interact or share information with the CRN terminals. Even though there exists spectrum sensing techniques that exploit the bidirectional nature of some primary networks or the weak leakage power of primary receiver RF circuits to infer the existence or the absence of a neighboring primary receiver [7], such schemes do not provide a way to measure the cumulative interference at the primary receiver.

The common feature of all the above spectrum sensing techniques is that they only consider one form or another of the conventional listen-before-talk (LBT) strategy to detect primary transmitters. LBT strategies typically has the CR continually listening to the different spectrum bands in order to asses the availability of spectral opportunities. While the $\mathrm{CR}$ is not actually receiving data during the idle listening process, it still consumes power that is comparable to the power consumed during data reception. Since idle listening used in LBT schemes is continually run by the CR over the spectrum bands of interest, it significantly contributes to the energy consumption of the CR. Extensive measurements in [8] have shown that $60 \%$ of the energy consumed in realworld wireless technologies that use LBT is consumed in idle listening, even with the use of power saving modes. In order to reduce the energy consumption of CRs, LBT needs to be revisited to allow the $\mathrm{CR}$ to identify the spectral opportunities based on the liminal amount of non-beneficial idle listening.

It is worth mentioning that the finite sensing duration of CR also limits the accuracy of spectrum sensing. Given a limited sensing period, only a certain sensing accuracy can be guaranteed. As the sensing duration increases, the reliability of the sensing information might increase. However, longer spectrum sensing windows are not necessarily useful since the environment is dynamic and the energy on a given channel is modulated both by the bursty traffic, asynchronous initiation and termination of packet transmissions, and channel fading.

\section{Spectrum Coordination Messaging $\mathrm{CO}_{2}$ Emission}

In multiuser CRNs, the coordination between multiple CR users is a major challenge not only from a networking point of view but also from energy consumption perspective. If legacy medium access control (MAC) protocols designed for traditional networks were to be used in CRNs, all CR users that infer a spectral opportunity will greedily attempt to exploit the sensed opportunity. Recall that legacy MACs often adopt greedy strategies that try to best utilize a channel access (e.g., by using the highest transmission rate or choosing the best channel). Such greedy approaches deteriorate the goodput of the CRN as the number of CR users increase due to increased blocking probability [4]. Furthermore, such greedy MACs are 


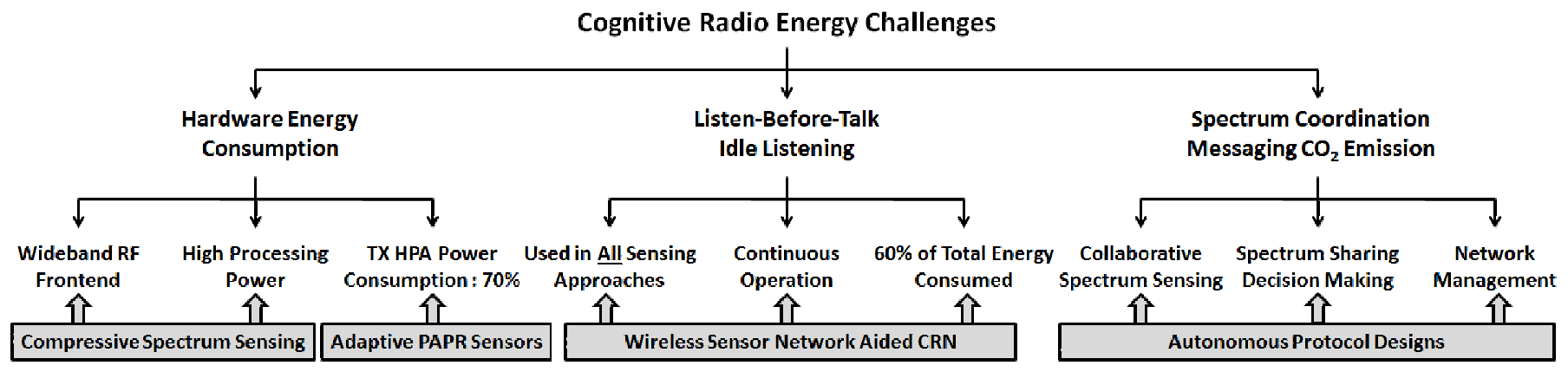

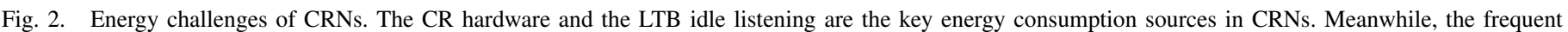

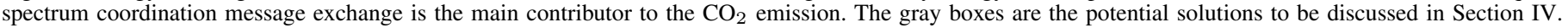

known to suffer from unfairness problems that may cause some cognitive sender-receiver pairs to dominate other pairs.

Several centralized and distributed cooperative MAC approaches have been recently developed for CRNs [9]-[11]. However, such schemes rely on the explicit coordination between different transmissions which is a main challenge in CRNs as it requires gathering and distributing spectrum information across the $\mathrm{CRN}$ and/or synchronizing the activities of different flows. The use of a common control channel for inter-flow coordination (as well as for the coordination between a sender and its respective receiver) makes it the bottleneck of a CRN and the single point of failure for the entire system [4].

Furthermore, the exchange of spectrum coordination messages significantly contributes to the $\mathrm{CO}_{2}$ emission of CRNs. This is due to the massive number of messages exchanged in order to collect the spectrum information from different $\mathrm{CR}$ terminals and then to distribute the joint spectrum access discission among them. In order to reduce the amount of $\mathrm{CO}_{2}$ emission of CRNs, spectrum sharing and access algorithms should minimize the explicit information sharing by either relying on local decisions as much as possible or exploiting learning and prediction techniques.

\section{Green Cognitive Radio Networks}

In this section, we overview the enabling technologies that tackle the above problems in the move towards green CRN.

\section{A. Compressive Spectrum Sensing}

Even though there is a significant research and industry effort in realizing wideband frontends with multiple $\mathrm{GHz}$ operating bandwidth, such wideband transceivers are expensive/nonpractical given today's technologies. Furthermore, spectrum sensing based on such wideband frontends typically uses traditional transformation techniques - such as wavelet transforms - to extract the spectrum utilization information from the wideband signal. Such wideband techniques sample the wideband signals at the Nyquist rate. Thus, they do not only suffer from the high complexity of acquiring such a large amount of samples, but also, the time needed to process such many samples to retrieve the spectrum information is large.

On the other hand, existing CR terminals use spectrum sensing techniques such as energy and waveform detectors developed for narrowband frontends [6]. In order to scan a wide frequency band of interest using a narrowband frontend, a bandpass filter is tuned to narrow frequency band. After sensing a given band, the CR sequentially senses all other bands. Such an approach does not only induce long delays in the retrieval of the sensing information but also is powerhungry. In order to reduce the sensing time, [12] uses a filter bank that is simultaneously tuned to several narrow sub bands. However, such an approach consumes more power hungry.

Compressive sensing provides a green solution to the energy consumption of spectrum sensing [13]. Compressive sensing was recently introduced in 2006 as a low-complexity techniques for acquiring the information content of wideband signals using sampling rates much lower than the Nyquist rate [14], [15]. Compressive sensing provides a generic way to compress a sparse signal without prior knowledge about the signal itself. If the acquired wideband signal is sparse enough in the frequency domain, only few random samples are enough to reconstruct the entire information content of the wideband signal. Consequently, the computational complexity of compressive sensing is significantly less than the computational complexity of the traditional transform coding techniques. Another advantage of compressive sensing is that information retrieval simultaneously takes places with the sampling process, i.e., there is no delay in information retrieval. Thus motivated, Tian and Giannakis proposed to use the compressive sensing theory to sense wideband spectrum in [13]. Serval subsequent approaches have been proposed since then (see [16] and the references therein). However, compressive spectrum sensing is still an open research problem since existing approaches relax the sparsity requirement of the wideband spectrum to be sensed. New algorithms are needed for densely occupied wideband spectrum, dealing with weak received signal levels, and realized via real-time algorithms.

\section{B. Wireless Sensor Network Aided CRNs}

Wireless Sensor Network (WSN) aided cognitive radio networking [17] combines the best of two worlds: it allows the CRN to exploit the spectral opportunities without paying the high energy price of CR spectrum sensing. Meanwhile, it takes advantage of the energy efficiency of WSNs while obtaining the spectrum information needed by the CRN. The use of a dedicated WSN for spectrum sensing does not only significantly reduce the energy consumption of the CR terminals but also improves the system's ability to identify the spectral opportunities compared to existing CR solutions [6]. There are two main architectures for WSN-aided CRNs [18]:

1) Independent sensor network aided CRN architectures: In this architecture, the WSN is deployed in an area by an operator and the cognitive radio users entering the area can communicate with the sensor network to get information about spectrum usage and/or instructions of how they can 
use the spectrum. Such an architecture guarantees that the primary transmitters will be detected with a defined probability regardless of the number of cognitive radios present in a given area. Such an architecture can exploit the energy efficiency of WSN to its full potential while reducing the CR hardware complexity, and hence, its power consumption.

2) Integrated sensor network aided CRN architectures: There does not exist an independent WSN in this architecture. Instead, the sensors are integrated in the CR user terminals. These sensors perform the sensing process and detect the available transmission opportunities in a collaborative manner. In this case, the cognitive network is also the wireless sensor network. In this architecture, only local sensing information in the areas where the CR users are located is obtained. Hence, the sensing accuracy increases with the number of CR users.

It is worth noting that the above two architectures can also be combined into a hybrid architecture as the case with the SEnsor Network for Dynamic and cOgnitive Radio Access (SENDORA) project [17]. The SENDORA project aims at providing mobile broadband services in the European Union countries at a low cost. The flexibility of a hybrid architecture allows to tradeoff the spectrum sensing accuracy with the energy efficiency, CR hardware complexity, network cost, etc.

\section{Autonomous Protocol Designs}

Existing spectrum management schemes developed for both centralized CRNs [19] and distributed CRNs [11] rely on collecting the spectrum measurements from different $\mathrm{CR}$ terminals and use them to make the spectrum access decisions for different transmissions. Basically, decision-theoretic approach and cooperative and non-cooperative games have been used in such spectrum management schemes. However, such techniques are associated with a significant spectrum coordination messaging overhead that contributes to the $\mathrm{CO}_{2}$ emission of CRNs as discussed in Section III.

Recently, learning techniques have also been used to identify a subset of the spectrum to sense and to find the optimal resource allocation (time, spectrum, and power) that maximizes the CRN goodput [20], [21]. In [20], two distributed cooperative learning and allocation schemes were proposed: one that assumes minimal prior knowledge of the CR information and the other does not assume such information. The objective of both schemes is to minimize the total regret in distributed learning (equivalently maximize the CRN goodput). Similarly, [21] maximizes the CRN goodput via resource allocation based on adaptive learning in spectrum probing.

In order to move towards green cognitive networks, new autonomous spectrum management protocols need to be developed. Such protocols should allow the CR nodes to (1) locally make accurate decision regarding the spectrum utilization, (2) individually adjust their transmission policies in order to fairly share the spectral opportunities with other CR terminals in the CRN. As a first step in this direction, we proposed and overhead-free spectrum management scheme in [22] and empirically demonstrated its performance in [23].

\section{CONCLUSIONS}

This paper has presented an overview of the paradigm shift towards green CRNs. Unlike traditional CRNs, green CRNs are aware of sustainable development, and improve the spectrum utilization efficiency via spectrum awareness at low energy consumption and reduced $\mathrm{CO}_{2}$ emission to the environment.
We have identified the main sources of energy consumption in CRs and key contributors to the $\mathrm{CO}_{2}$ emissions in CRNs. Then, we presented an overview of the major techniques that can lead the move towards the green cognitive radio vision, more specifically, compressive spectrum sensing, WSN-aided $\mathrm{CRN}$, and autonomous CRN protocol designs.

\section{REFERENCES}

[1] J. Palicot, "Cognitive radio: an enabling technology for the green radio communications concept," in Proc. of IEEE IWCMC'09, 2009.

[2] J. Palicot and H. Zhang, "Cognitive radio for green communications and networking," in IARIA AICT 2013, Jun. 2013.

[3] G. Gur and F. Alagoz, "Green wireless communications via cognitive dimension: an overview," IEEE Network, vol. 25, no. 2, 2011.

[4] I. F. Akyildiz, W.-Y. Lee, and K. R. Chowdhury, "CRAHNs: Cognitive radio ad hoc networks," Ad Hoc Networks (Elsevier), vol. 7, no. 5, 2009.

[5] J. Mitola III, "Cognitive radio: An integrated agent architecture for software defined radio," Ph.D. dissertation, KTH Royal Institute of Technology, 2000.

[6] T. Yucek and H. Arslan, "A survey of spectrum sensing algorithms for cognitive radio applications," IEEE Communications Surveys and Tutorials, vol. 11, no. 1, pp. 116-130, First Quarter 2009.

[7] C.-H. Lee and M. Haenggi, "Delay analysis of spatio-temporal channel access for cognitive networks," in Proc. of IEEE ICC 2011, Kyoto, Japan, Jun. 2011.

[8] X. Zhang and K. G. Shin, "E-MiLi: Energy-minimizing idle listening in wireless networks," IEEE Trans. on Mob. Comp., vol. 11, no. 9, 2012.

[9] Q. Zhao, L. Tong, A. Swami, and Y. Chen, "Decentralized cognitive MAC for opportunistic spectrum access in ad hoc networks: a POMPD framework," IEEE Journal of Selected Areas in Communications, vol. 25, no. 3, pp. 589-600, Apr. 2007.

[10] F. Wang, M. Krunz, and S. Cui, "Price-based spectrum management in cognitive radio networks," IEEE Journal of Selected Topics in Signal Processing, vol. 2, no. 1, pp. 74-87, Feb. 2008.

[11] H. B. Salameh, M. Krunz, , and O. Younis, "MAC protocol for opportunistic cognitive radio networks with soft guarantees," IEEE Trans. on Mobile Computing, vol. 8, no. 10, pp. 1339-1352, Oct. 2009.

[12] B. F. Boroujeny, "Filter band spectrum sensing for cognitive radios," IEEE Transactions on Signal Processing, vol. 56, no. 2, 2008

[13] Z. Tian and G. Giannakis, "Compressed sensing for wideband cognitive radios," in In Proc. of IEEE ICASSP, Honolulu, HI, Apr. 2007.

[14] E. J. Candés, "Compressive sampling," in Proc. of International Congress of Mathematicians, Madrid, Spain, Aug. 2006.

[15] R. Baraniuk, "Compressive sensing," IEEE Signal Processing Magazine, vol. 24, no. 4, 2007.

[16] H. Sun, A. Nallanathan, C.-X. Wang, and Y. Chen, "Wideband spectrum sensing for cognitive radio networks: a survey," IEEE Wireless Communications, vol. 20, no. 2, pp. 74-81, 2013.

[17] "SENDORA: SEnsor Network for Dynamic and cOgnitive Radio Access." [Online]. Available: http://www.sendora.eu/

[18] C. Bonnet, D. Camara, R. Ghaddab, L. Iacobelli, F. Kaltenberger, R. Knopp, B. Mercier, N. Nikaein, D. Nussbaum, E. Yilmaz, and B. Zayen, "Sensor network aided agile spectrum access through lowlatency multi-band communications," in IEEE DCOSS'11, Jun. 2011.

[19] C. Raman, R. D. Yates, and N. B. Mandayam, "Scheduling variable rate links via a spectrum server," in Proc. of the IEEE DySPAN 2005, Baltimore, MD, Nov. 2005.

[20] A. Anandkumar, N. Michael, and A. Tang, "Opportunistic Spectrum Access with Multiple Users: Learning under Competition," in Proc. of IEEE INFOCOM 2010, San Deigo, USA, Mar. 2010.

[21] P. Chaporkar, A. Proutiere, and H. Asnani, "Learning to optimally exploit multi-channel diversity in wireless systems," in Proc. of the IEEE INFOCOM 2010, San Diego, CA, Mar. 2010.

[22] A. Khattab, D. Perkins, and M. A. Bayoumi, "Rate-adaptive probabilistic spectrum management for cognitive radio networks," in Proc. of the IEEE WoWMoM 2011, Lucca, Italy, Jun. 2011.

[23] A. Khattab, D. Perkins, and M. Bayoumi, "Design, implementation and characterization of practical distributed cognitive radio networks," IEEE Transactions on Communications, vol. 61, no. 10, pp. 4139-4150, Oct. 2013 